\title{
Solar Neutron and Gamma-ray Monitor for a Small Satellite
}

\section{Kazutaka Yamaoka* for the ChubuSat-2 collaboration}

Division of Particle and Astronomy, Nagoya University, Furo-cho, Chikusa-ku, Nagoya 464-8601

E-mail: vamaokadisee.nagova-u.acjp

\begin{abstract}
Solar neutrons can be probles for clarifying the ion acceleration mechansm in the Sun. However, there are only a few 10 detections up to present. The SEDA-AP Fiber detctor on the International Space Station (ISS) has been currently observing solar neutrons from space. In order to proceed solar-flare studies, we have developed very compact solar neutron and gamma-ray monitor for the micro satellite ChubuSat-2 utilizing the multi-pixel photon counter (MPPC) as a readout of scintillators. The realized size of the detector is $15 \mathrm{~cm} \times 17 \mathrm{~cm} \times 18.5 \mathrm{~cm}$ and its weight is $6.2 \mathrm{~kg}$. We launched it on February 2016, but the detector has not been turned on yet due to a trouble in the satellite bus system. To upgrade the detector, we will use an ASIC instead of discrete electric parts for MPPC readout to reduce the electric power and space. In this paper, we will describe details of solar neutron detectors on the ChubuSat-2 and a future satellite.
\end{abstract}

35th International Cosmic Ray Conference -ICRC2017-

10-20 July, 2017

Bexco, Busan, Korea

${ }^{*}$ Speaker. 


\section{Introduction}

Solar flares are explosions caused by magnetic energy release via magnetic reconection process (e.g. [四). Many observations of solar flares have been carried out through electromagnetic waves such as optical, ultra-violet and X-rays and charged particles from space and on the ground, but ion acccerelation mechanisms in solar flares are still unresolved problem. Several models such as shock and statistical acceleration have been proposed. Neutrons are not affected by the magnetic field on the solar surface and in the interstellar medium unlike protons and electrons, and thus they reach the Earth with original information about acceleration on the solar surface maintained. Hence they are considered to be direct probes to clarify the ion acceleration mechanisms.

An expected solar neutron flux was originally discussed by Biermann et al. (1951) [వ]. Solar neutrons were first observed by Solar Maximum Mission (SMM) in 1980 [ [ ] ], but there have only been about a few tens of successful detections so far. Solar neutron observations have been mainly carried out from not space but the ground [ [ 4 ]. The currently working space detector, SEDAAP FiBer detector (FIB), [可] on the international space station (ISS), was affected by the secondary neutron background due to the ISS itself with a huge mass ( $\sim 400$ ton). A small satellite is expected to have a much smaller neutron background than that of SEDA-AP. Furthermore, neutron detectors in space can access energies lower than $100 \mathrm{MeV}$. Neutrons are attenuated by the Earth's atmosphere (attenuation factor: 1/10000-1/100 depending on the altitude), so it is difficult to observe neutrons with an energy below $100 \mathrm{MeV}$ on the ground.

This solar neutron observation project using micro satellite was originally proposed by graduate students at Nagoya University. Nagoya university started a leadership development program for space exploration and research as a program for the leading graduate school (LGS), [⿴囗6 since FY 2012 after selection by Japan Society for the Promotion of Science (JSPS). This program aims to develop international leaders who can spur innovations that will expand the space utilization using the ChubuSat development. One group consisting of six graduate students with different research fields and backgrounds proposed this unique mission in the semester of FY 2014, and the proposal is highly rated in this program.

For this purpose, we constructed a radiation detector (RD) [ $[\mathbb{Z}]$ which can detect solar neutrons for $50-\mathrm{kg}$ class satellite ChubuSat-2. This detector can also detect gamma-rays as the Compton camera technique. ChubuSat is a series of 50-kg class microsatellite developed by Nagoya university, Daido university, and small or medium-sized aerospace companies (Monozukuri Aerospace Support Techonology Team: MASTT) around the Chubu region in Japan, and the second ChubuSat, ChubuSat-2, was selected as one of the four piggy-back satellites of Hitomi (ASTRO-H) mission [8] by JAXA in summer 2014. We already launched the ChubuSat-2 satellite with Hitomi (ASTROH) mission on February 16, 2016. Unfortunatelly the RD has not been turned on due to troubles in the power control unit. In this paper, we will report on cocept and development of the RD onboard the ChubuSat-2 satellite, and describe future prospects for development of similar types of detectors for a small satellite. 


\section{Design of the Radiation Detector (RD)}

\subsection{Sensor}

Our detector concept for neutron detections is based on the design of SEDA-AP and groundbased neutron telescopes. The detector structure is shown in Figure $\mathbb{W}$. The detector consists of the three parts: neuron detection, gamma-ray detection, and anti-coincidence parts. The neutron detection part is composed of an array of plastic scnillarot bars of $1 \mathrm{~cm} \times 1 \mathrm{~cm} \times 10 \mathrm{~cm}$. 10 layers. A neutron interacts with hydrogen atoms in the plastic scintillator bars via elastic scattering. The recoil proton loses its energy in each bar via ionization loss passing through some bars. The incident neutron energy in the 30-120 MeV range can be determined by measuring energies and direction of recoiled protons. Our neutron detecor is also sensitive to soft gamma-rays in the 200-1000 $\mathrm{keV}$ range with the Compton camera technique. A gamma-ray interacts with the plastic scintillator by Compton scattering, and a scattered photon is absorbed in the GAGG (Ce: $\mathrm{Gd}_{3} \mathrm{Al}_{2} \mathrm{Ga}_{3} \mathrm{O}_{12}$ ) inorganic scintillator array placed on the bottom part of the detector. Using two interacting points in the plastic and GAGG scintillator, we can determine the incident energy of a gamma-ray and constrain its incident angle to an arc within $\sim 10$ degrees. By accumulating some photon events coming from the same direction, we can identify a gamma-ray source (this is the "Compton camera"). We can also use only a photoelectric absorption event in the GAGG scintillator array after passing through plastic scintillators. We also put the anti-coincidence detector which surrounds the plastic scintillor array to reject charged partciles efficiently. The anti-coincidence detector is made by plastic scintillator plate with $0.8 \mathrm{~cm}$ thinckness.

In order to realize this detector for a small satellite, we utilize a novel silicon semiconductor device to read out scintillation signals: Silicon Photomultiplier (or Multi Pixel Photon Counter (MPPC)) developed by Hamamatsu Photonics. It has a sensitive area of $6 \times 6 \mathrm{~mm}$ and a aquantum efficiency of $35 \%$ at $450 \mathrm{~nm}$. It uses a very low bias voltage, $50-60 \mathrm{~V}$, and can realize a high gain of $10^{6}$ which is similar to that of a photo-multiplier tube (PMT) with a high bias voltage $(\sim 1000 \mathrm{~V})$. The GAGG scintillator is also relatively new devince among scintillators. It has a higher light output (48000 photons/MeV) and faster reponse (decay time $88 \mathrm{~ns}$ ) than those of $\mathrm{NaI}(\mathrm{Tl})$, and is mechanically stable around room temperature, Both MPPC and GAGG scintillator are very attractive devices with an excellent performance as radiation sensors, but have not been used in space yet. Hence, verification of the detector technology is also another purpose of this instrument.

\subsection{Electronics}

The schematic view of the signal processing electronics is shown in Fig. \. Each plastic scintillator bar is read out with MPPCs at both sides to determine the incident position along the bar direction, and 2 MPPCs are attached to one anti-coincidence detector. The GAGG $10 \times 10$ array is read out by MPPCs attached to each GAGG crystal. So there are in total 312 channels consisting of 200 from plastic bars, 12 from anti detectors, and 100 from the GAGG crystal array. The MPPC signals are processed independently by analog and digital electronics boards located around or under the sensors. The charge from each MPPC is sent to an electrical board and converted into a voltage pulse in the fast charge-sensitive pre-amplifier, and after amplification, the pulse height $(\mathrm{PH})$ is converted to a digital value by a 14-bit analog digital converter (ADC) with sample and hold 


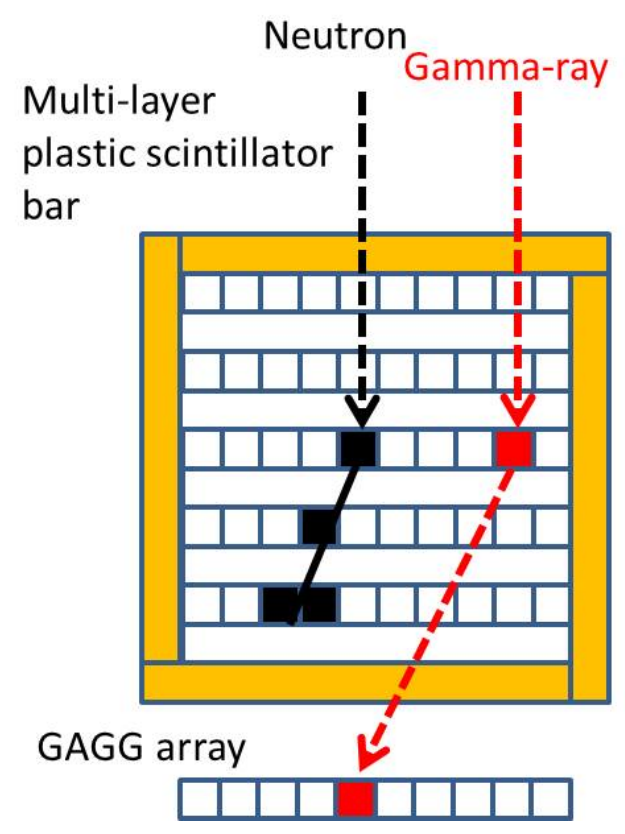

Figure 1: Detection principle of the RD. Neutrons can be detected in multi-layered plastic scintillators (shown by black), while gamma-rays can be detected in both plastic and GAGG scintillators via Compton scattering (shown by red). The anti-coincidence detectors (shown by orange) are used for rejection of charged particles such as protons and electrons.

function at the pulse peak timing. A high voltage $+54 \sim 55 \mathrm{~V}$ is required for the MPPC operation, so the electronics board provides the bias voltage to each sensor. This voltage is controlled and adjusted by checking the sensor temperature automatically in a Field-Programmable Gate Array (FPGA).

When an event trigger occurs in any plastic scintillator bar or any GAGG crystal (trigger is selectable), the data acquisition will start. The event-by-event data with all the PHs from 312 channels are produced with a size of $\sim 600$ byte per event ( 2 bytes per channel). Since the original data size is relatively large and the down-linked data size is limited to about 10 Mbyte per day, the RD data will have to be compressed by the on-board FPGA. Anti-coincidence events are judged as charged particles, and have to be removed. In addition, we implemented several logics: 1)removal of the detector channels with a low PH value (so-called zero-suppression) and 2)reduction of the trigger rate by a factor of $1 / n(n=1,2,4, \ldots .128)$ in the nominal data and change of the reduction rate during a solar flare where the RD detects a rate increase automatically. In this compression, the data size will be reduced by a factor of about 10, and compressed data are sent to the OnBoard Compturer (OBC) and stored in the OBC on-board memory with a size of 1 Gbyte until the telemetry down-link starts via the $\mathrm{S}$ band. We also have a housekeeping (HK) data for monitoring the RD power status, high voltage values and temperatures, which are outputted at fixed time intervals to the $\mathrm{OBC}$. 


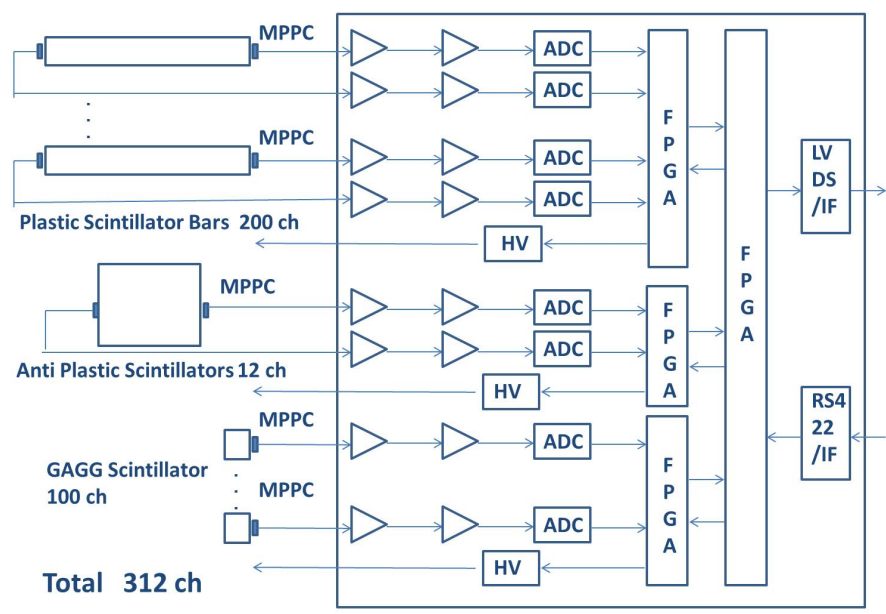

Figure 2: Signal processing for the RD. The signals from 312 MPPCs are processed independently by electronics. The mission data from the RD outputs to the on-board computer (OBC) while the OBC issues commands from the ground station to the RD.

\section{Development and Performance of the RD}

Based on the detector concept, the RD has been newly designed and developed by Nagoya University in collaboration with Clear Pulse Co., Ltd. The detector is mainly composed of sensors and electronics for signal processing from sensors. To make the sensor size large and the detector compact, electronics boards surround sensors (see right panel of Figure (3)). All the components are installed in one Aluminum box with a size of $15.2 \mathrm{~cm} \times 17.0 \mathrm{~cm} \times 18.5 \mathrm{~cm}$. The weight is $6.2 \mathrm{~kg}$ and the total power consumption is about $12 \mathrm{~W}$ during nominal operation. It is covered by multilayer insulator (MLI) to avoid thermal input from surrounding bus instruments (see left panel of Figure (B]). The RD is attached to $+Y$ panel just behind the solar panel, and thermally coupled to the radiator which is made of carbon graphite sheets [9]. Due to such passive thermal control, the RD temperature is designed to kept as low as possible to reduce the thermal noise of the semi-conductor devices. The specification of the detector is shown in Table $\mathbb{W}$.

After the detector fabrication, we also performed verification test, thermal vacuum and vibration test. The detector performance was checked using cosmic-ray muons and radio isotopes on the ground during pre-flight performance test. We found that most of all sensors worked well and do not change after the thermal vaccumm and vibration tests. The muon events were clearly detected by the RD, as shown in Figure $\mathbb{A}$, and photo peaks at $662 \mathrm{keV}$ gamma-rays from radio-isotope ${ }^{137} \mathrm{Cs}$ were visible in 99 sensors of the GAGG array.

The Chubusat- 2 was sucessfully launched by H-IIA launch vehicle \#30 at JAXA Tanegashima Space Center on February 12, 2016. The communication between satellite and ground station via amateur radio band has been well established soon after the launch. However, due to the trouble in interface between power control unit (PCU) and OBC, the PCU have not provided a power supply to the RD yet. The satellite is still in orbit and is accessible via amateur band, but it would be difficult to recover the satellite in a future.

To recover the solar science which should be achieved by the ChubuSat- 2 mission, we plan 
to upgrade the RD by making it more compact and reducing the electrical power. One important revision is to replace the elecric discrete parts such as operational amplifiers and discriminators with an Analog Specified Integrated Circuit (ASIC) which is developed and provided by IDEAS. This ASIC can process 16 signals from MPPCs independently with a very low power consumption of $15 \mathrm{~mW}$. We are now testing a basic performance of scintillators by readout them with the ASIC.

Table 1: Specifications of the radiation detector.

\begin{tabular}{l|l}
\hline \hline Detector & $\begin{array}{l}\text { Upper part: 10 plastic scintillator bars } \times 10 \text { layers } \\
\text { Lower part: GAGG }(\mathrm{Ce}) 10 \times 10 \text { scintillator array } \\
\text { read out with Multi Pixel Photon Counters }\end{array}$ \\
Number of Processing Signals & $\begin{array}{l}\text { Total: } 312 \text { (Plastic scintillar bar for both sides: 200, } \\
\text { Anti-coincidence detector (2 per face) : 12, GAGG array: } 100 \\
100 \mathrm{~cm}^{2}\end{array}$ \\
Geometrical Area & $30-120 \mathrm{MeV}$ (Neutrons) \\
Energy Range & $200-1000 \mathrm{keV}($ Gamma-rays) \\
& $1 \sim 2 \%$ (Neutrons) \\
Detection Efficiency & $1 \sim 2 \%($ Gamma-rays) \\
& $6.2 \mathrm{~kg}$ \\
\hline Weight & $15.2 \mathrm{~cm} \times 17.0 \mathrm{~cm} \times 18.5 \mathrm{~cm}$ \\
Size & $12 \mathrm{~W}$ \\
Nominal Power Consumption & $1 \mathrm{Gbyte}$ \\
Onboard Data Memory & $\sim 5 \mathrm{Mbyte}$ per day \\
Down-linked Data Size &
\end{tabular}
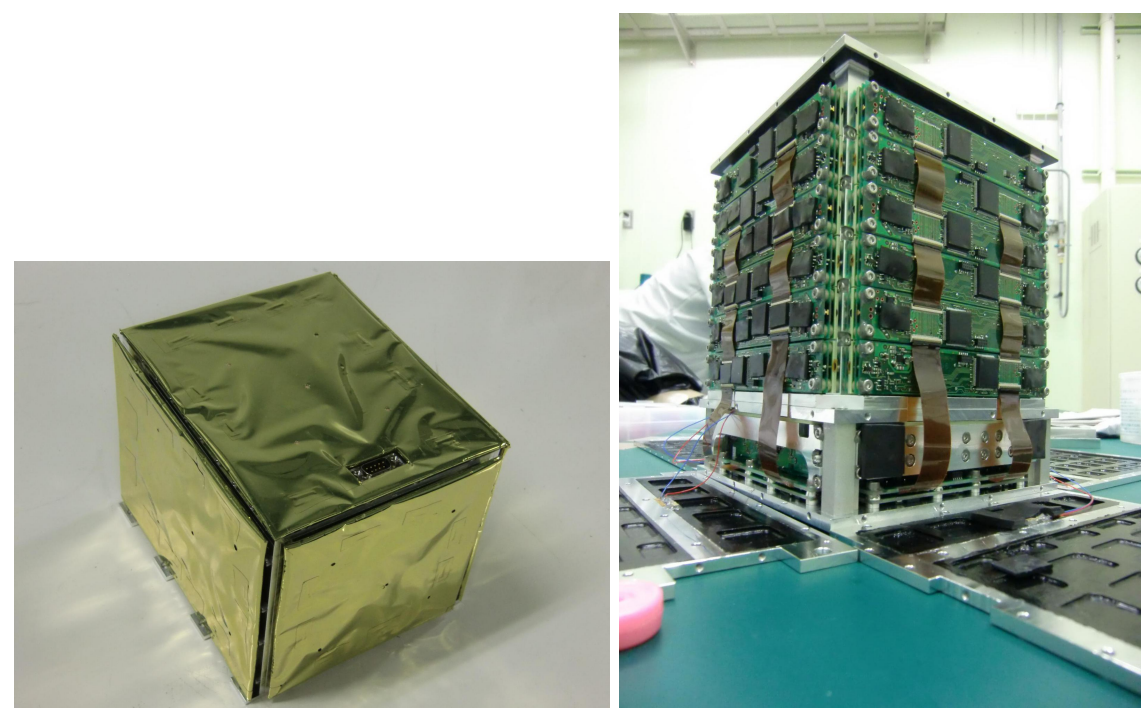

Figure 3: Left: Radiation detector (RD) onboard the ChubuSat-2 satellite. The detector is covered by MLI to keep its tempeture low. Right: RD internal structure. The sensors are covered by electronics boards. 

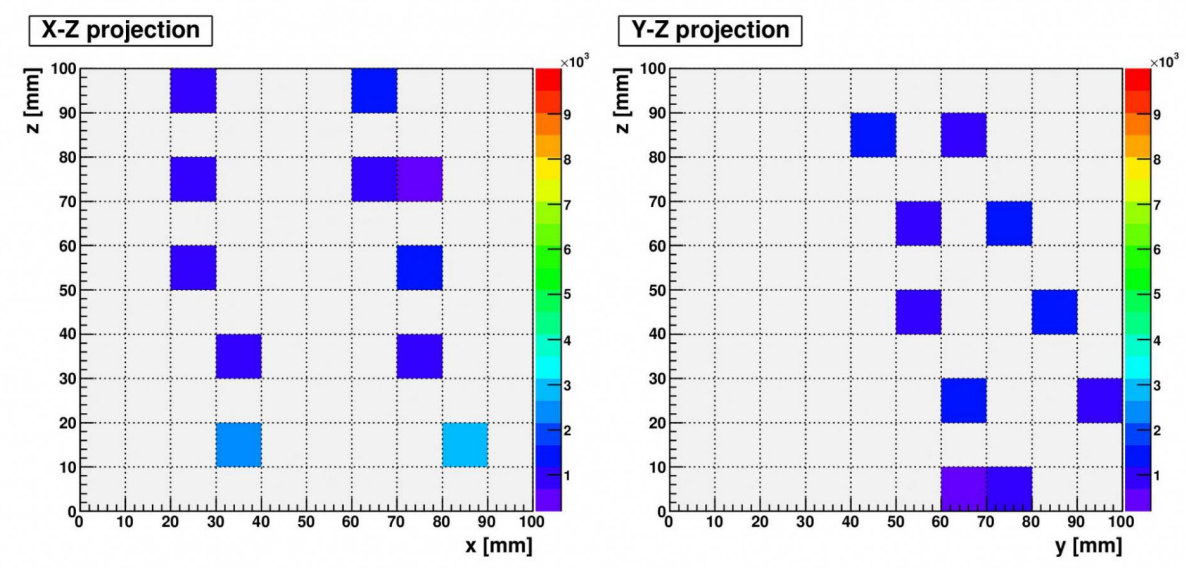

Figure 4: Cosmic-ray muon track (X-Z and Y-Z plane) in the RD during pre-flight performance test. Two muon events were simultaneously detected.

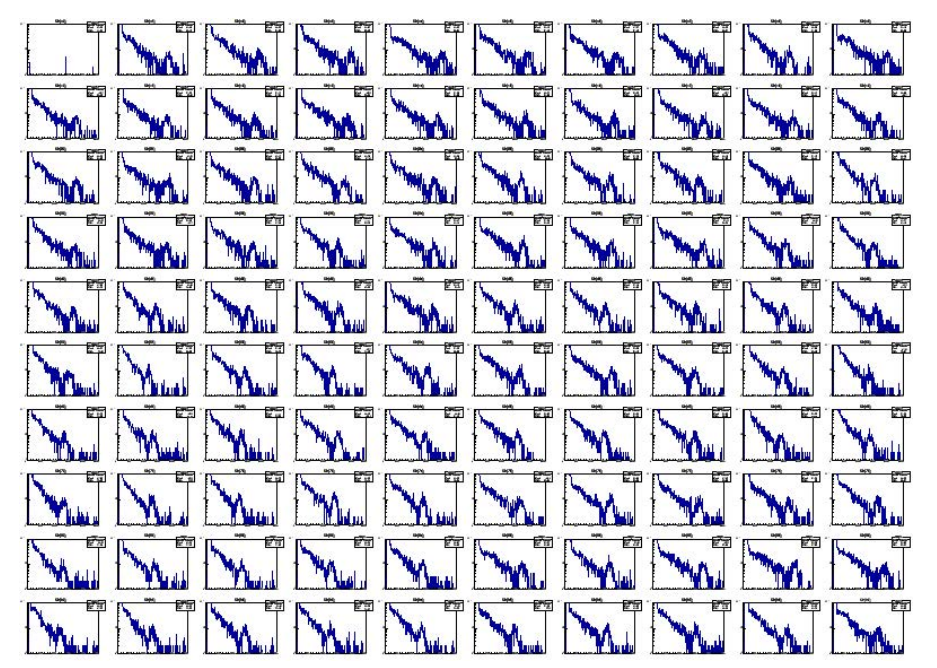

Figure 5: ${ }^{137}$ Cs spectra from GAGG $10 \times 10$ array. The $662 \mathrm{keV}$ photo-peak was clearly visible in most of the GAGG sensors. 


\section{Summary}

We have designed and developed novel solar neutron and gamma-ray detector for 50-kg class ChubuSat-2 satellite. This compact detector was realized by utilizing a new semi-conductor device SiPM (MPPC). The ChubuSat-2 satellite was succesfully launched in February 2016, but it has not been turned on yet. We are currenly tring to upgrade this type of detector by replacing electic parts with ASIC, and hope to recover this mission for a future small satellite to exploring new solar science.

\section{References}

[1] Shibata, K. \& Magara, T., Solar Flares: Magnetohydrodynamic Processes, Living Rev. Sol. Phys. (2011) 8: 6

[2] Biermann, L., Haxel, Ol, chuluter, A., Neutral cosmic rays produced in the Sun, Z Naturforsch A, 6(1) (1951):47-48

[3] Chupp, E. L. et al., A Direct Observation of Solar Neutrons Following the 0118 UT Flare on 1980 June 21, Astrophysical Journal, 263(1982) pp. L95-L98.

[4] Sasai, Y. et al., Performance of the SciBar Cosmic Ray Telescope (SciCRT) toward the Detection of High-Energy Solar Neutrons in Solar Cycle 24, Earth, Planet and Space, 66 (2014), article number 130.

[5] Muraki, Y., Koga, K., Goka, T., Matsumoto, H., Obara, T., Okudaira, O., Shibata, S., Yamamoto, T., Measurement by FIB on the ISS: Two Emissions of Solar Neutrons Detected?, Advances in Astronomy, (2012), article ID 379304.

[6] Leadership Development Program for Space Exploration and Research, Nagoya University Program for Leading Graduate Schools (LGS) web site: http://www.frontier.phys.nagoya-u.ac.jp/index-e.html (cited 1 Jan. 2012).

[7] Yamaoka, K. et al., Solar Neutron and Gamma-ray Monitor on the ChubuSat-2 Satellite, Trans. of the Japan society for aeronautical and space sciences, aesospace technology japan, Vol. 14 (2016) No. ists30 p. Pf_141-Pf_146

[8] Takahashi, T. et al.: The ASTRO-H X-ray Astronomy Satellite, Proceedings of the SPIE Astronomical Instrumentation "Space Telescopes and Instrumentation 2014: Ultraviolet to Gamma Ray”, 9144 (2014) 914425-1-914425-24.

[9] Park, D., Miyata, K., \& Nagano, H., Thermal design and validation of radiation detector for the ChubuSat-2 micro-satellite with high-thermal-conductive graphite sheets, Acta Astronautica, Volume 136 (2017), p. 387-394. 